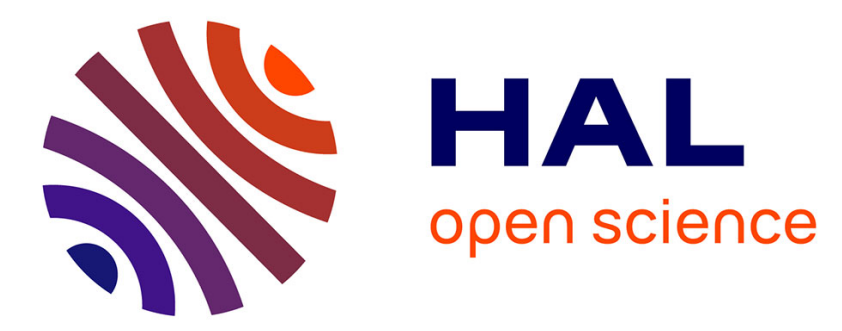

\title{
Penser les négociations collectives pour penser l'entreprise. Introduction
}

Claude Didry, Camille Dupuy, Denis Giordano, Jules Simha

\section{To cite this version:}

Claude Didry, Camille Dupuy, Denis Giordano, Jules Simha. Penser les négociations collectives pour penser l'entreprise. Introduction. Négociations, 2021, n 35, pp.9-22. 10.3917/neg.035.0009 . halshs03506468

\section{HAL Id: halshs-03506468 \\ https://shs.hal.science/halshs-03506468}

Submitted on 2 Jan 2022

HAL is a multi-disciplinary open access archive for the deposit and dissemination of scientific research documents, whether they are published or not. The documents may come from teaching and research institutions in France or abroad, or from public or private research centers.
L'archive ouverte pluridisciplinaire HAL, est destinée au dépôt et à la diffusion de documents scientifiques de niveau recherche, publiés ou non, émanant des établissements d'enseignement et de recherche français ou étrangers, des laboratoires publics ou privés. 


\title{
Penser les négociations collectives pour penser l'entreprise. Introduction.
}

\author{
Claude Didry, CNRS, ENS, $\mathrm{CMH}^{1}$
}

Camille Dupuy, Université Rouen Normandie, DySoLab, CEET ${ }^{2}$

Denis Giordano, CNRS, ENS, $\mathrm{CMH}^{3}$

Jules Simha, Université Paris, Cerlis, CEET ${ }^{4}$

Si le travail et son organisation subissent indéniablement des transformations en lien avec le développement des outils numériques (Benedetto-Meyer, Boboc, 2021), cette focalisation tend à faire oublier que celui-ci continue à se réaliser massivement au sein d'emplois salariés exercés au sein d'entreprises. Ce sont ainsi les entreprises qui ont été au cœur des plans de relance économique conduits à travers le monde dans le contexte de la crise économique qui a accompagné la crise sanitaire du covid-19, en laissant fréquemment dans l'ombre les enjeux de la relance pour les salariés, leurs représentants et représentantes. De ce point de vue, l'entreprise apparaît comme un espace central de la vie économique et sociale, y compris dans le maintien d'un lien par-delà la distance requise par la lutte contre la pandémie.

Cette prégnance actuelle de l'entreprise fait écho aux travaux de Max Weber qui dès le début $\mathrm{du} \mathrm{XX}^{\mathrm{ème}}$ siècle fait de l'entreprise un concept fondamental de la sociologie. En la définissant comme « une action continue en finalité d'un genre déterminé », c'est-à-dire une organisation «dotée d'une équipe administrative agissant continûment en finalité » (Weber 2016 [1920], 158), il reconnaît d'emblée son caractère pluriel, à la fois unité productive (une action en finalité) et espace social (son équipe). Dans l'œuvre de Weber, la reconnaissance de ce caractère fondamental de l'entreprise coïncide avec l'avènement d'un capitalisme « moderne » remettant en cause un capitalisme «traditionnaliste » de négociants centralisant une production artisanale dispersée ${ }^{5}$. En effet, elle se constitue autour de la figure d'un capitaine d'industrie intégrant de manière durable des travailleurs et travailleuses à une activité collective de production qui caractérise l'avènement d'un capitalisme industriel (Didry, 2020).

Cette analyse trouve un écho, aux États-Unis, dans les travaux fondateurs de Ronald Coase (1937). Dépassant la théorie économique standard qui pense l'entreprise comme une unité de décision cherchant à maximiser son profit, cet auteur analyse la «nature de la firme ». Pour celui-ci, l'entreprise est le lieu d'une coordination par l'autorité tandis que le marché est le lieu d'une coordination par les prix. En caractérisant l'entreprise à travers les coûts de coordination entre employeur et salariés, il ouvre la voie à une analyse de l'entreprise comme un espace tant économique que social. Ses analyses, poursuivies par Oliver Williamson (1975), expliquent ainsi l'émergence de la grande entreprise par une efficacité supérieure à

\footnotetext{
1 claude.didry@ens.fr

2 camille.dupuy@univ-rouen.fr

3 giordano.denis@gmail.com

4 jules.simha@parisdescartes.fr

${ }^{5}$ Sur ce processus, voir Weber, 2003 [1905], p. 46-60.
} 
celle du marché en termes de «coûts de transaction». Cette conceptualisation partage avec l'analyse wébérienne l'idée d'une contingence de l'entreprise qui n'est pas donnée avec un capitalisme qui peut lui-même se concevoir à partir des interrelations marchandes entre une multitude d'entrepreneurs et de sous-entrepreneurs.

La reconnaissance de cette centralité conceptuelle de l'entreprise se retrouve, plus près de nous, dans le champ d'une sociologie de l'entreprise ouvert par l'œuvre pionnière de Renaud Sainsaulieu, pour qui l'entreprise ne se réduit pas à « un simple appareil de production «mais se présente comme «l'institution centrale d'une société » (1990, 15). L'entreprise apparaît ainsi tout à la fois organisation productive et institution sociale et juridique (Segrestin, 1996 ; Thuderoz, 1997).

\section{Définir l'entreprise : des cheminements menant à une impasse ?}

Pourtant, malgré l'intérêt qu'elle suscite dans les sciences sociales, la notion d' «entreprise » demeure floue : tout à la fois organisation visant l'efficience économique, communauté de travail dominée par la figure de l'employeur, œuvre collective et unité légale. Face à un tel flou, ce numéro spécial de Négociations propose d'explorer à nouveaux frais l'entreprise comme un fait social, en renvoyant aux pratiques concrètes et régulières d'une multitude d'acteurs (actionnaires, salariés, représentants des directions et du personnel) que désigne la négociation collective. Il s'agit ainsi, dans une perspective originale, d'analyser les contours de l'entreprise à partir des négociations collectives qui s'y déploient.

Pour les juristes, le terme d'entreprise, pourtant présent dans la langue du droit du travail et celle du droit commercial, ne désigne pas une réalité clairement identifiable. Ainsi, en France, « le droit ignore l'entreprise », tandis qu'il organise la «société » (Lyon-Caen, 2014, 24). Si l'entreprise détermine pour les salariés liés à un même employeur le droit à une représentation collective, sous la forme notamment du comité d'entreprise (Jeammaud, Kirat et Villeval, 1996), sa transformation en un comité social et économique (CSE) par les ordonnances dites «Macron » ${ }^{6}$ de 2017 contribue à entretenir le flou. À l'étranger, il arrive que le droit introduise des éléments de définition de l'entreprise, sans pourtant, là non plus, en fixer véritablement les traits. Par exemple, au Canada, la jurisprudence établit qu'une entreprise est « un ensemble organisé suffisant des moyens qui permettent substantiellement la poursuite en tout ou en partie d'activités précises » (affaire U.E.S., Local 298 c. Bibeault, 1988), tandis qu'en Belgique, le code de droit économique la définit comme «toute personne physique ou personne morale poursuivant de manière durable un but économique, y compris ses associations », sans plus de précisions sur sa nature. Par ailleurs, si la notion d'entreprise s'inscrit parfois dans un contour juridique au sein des droits nationaux, le développement de formes de plus en plus complexes et diverses d'organisations productives, sous la forme notamment de groupes intégrant un ensemble de sociétés - parfois à une échelle multinationale - soulève des questions sur la délimitation même de ce contour. Cela a conduit

\footnotetext{
${ }^{6}$ Ordonnance $\mathrm{n}^{\circ}$ 2017-1386 du 22 septembre 2017 relative à la nouvelle organisation du dialogue social et économique dans l'entreprise et favorisant l'exercice et la valorisation des responsabilités syndicales.
} 
la statistique publique en France à préciser, au-delà de ce cadrage juridique, cette notion. Prenant acte de ce développement de groupes agrégeant un ensemble plus ou moins large de sociétés, l'Institut national de la statistique française (INSEE) a ainsi revu sa définition de l'entreprise. Autrefois centrée sur les «unités légales» que constituent juridiquement les établissements et les sociétés, l'INSEE définit désormais l'entreprise comme «les regroupements économiquement pertinents d'unités légales appartenant à un même groupe de sociétés » (INSEE, 2019, 13). Les statisticiens se fondent pour cela sur le «profilage» d'entreprises économiquement autonomes dans l'affectation de leurs ressources - au-delà des « unités légales » qui les constituent - à travers les flux financiers entre celles-ci que prend en compte l'enquête LIFI (Liaisons Financières).

\section{Contourner l'impasse : définir l'entreprise via les pratiques de négociation}

Au-delà de l'enjeu juridique et statistique, ce sont à la fois les contours de l'entreprise mais aussi son épaisseur sociale qui interrogent. Il s'agit ainsi dans ce numéro spécial de saisir cette entité non seulement comme un périmètre qui regroupe des activités productives, mais également en tant que collectivité de travailleurs et travailleuses relevant d'une «société » voire d'un «établissement» qui agissent en vue d'une « entreprise » commune. Ces interrogations nous renvoient à celles d'Adolf Berle et Gardiner Means. Dès 1932, les auteurs dessinent les intérêts pluriels de la firme. S'ils insistent sur deux groupes, les actionnaires et managers, ils pointent également le rôle des salariés et des fournisseurs. Ils proposent ainsi une conception de l'entreprise comme une institution collective au service de la collectivité. Cette conception interroge sur la place de l'entreprise dans la société et fait largement écho à des préoccupations actuelles. Les accidents et les drames qui touchent cette collectivité suscitent en effet une explicitation de la responsabilité sociale de l'entreprise tant devant les tribunaux que face à l'opinion publique (Barraud de Lagerie, 2011). Les codes de conduite des multinationales annoncent des engagements publics de l'entreprise, en matière d'environnement, de corruption, de discrimination, dont la mise en œuvre revient aux salariés de celle-ci (Béthoux, Didry \& Mias 2007). Au sein de cette communauté, la figure de l'employeur est centrale même si elle peut être complexe et lointaine, comme dans le cas de sociétés contrôlées par des groupes. Dans celui des activités de plateformes, elle se dissout parfois en une multitude d'entrepreneurs, sous la forme de prestataires de services renvoyant tout autant à des personnes physiques (micro-entrepreneurs) qu'à des sociétés employant à leur tour des salariés (par exemple lorsque des sociétés confient leurs flottes de véhicules à des chauffeurs). Pour saisir alors ce qu'est l' «entreprise», de nombreux travaux en sociologie, économie, sciences de gestion, droit, sciences politiques ou histoire ont cherché à ouvrir la «boîte noire » de l'entreprise, qualifiée de «point aveugle du savoir » (Segrestin et al., 2014). C'est précisément dans cette perspective qu'il est proposé de «penser le travail pour penser l'entreprise » (Favereau et al., 2016), en mettant au jour l'importance, mais aussi l'insuffisance, des mécanismes de codétermination associant les travailleurs et travailleuses à la gestion de l'entreprise. 
Dans le même mouvement, ce numéro spécial propose d'élargir le regard en postulant que les négociations collectives constituent un ensemble de pratiques permettant à leur tour de caractériser l'entreprise. En tant qu' « être collectif » qui ne se réduit pas à une organisation marchande en quête de profit (Segrestin \& Hatchuel, 2012, 15), l'entreprise y est analysée à partir des collectifs de travail qu'elle agrège et des relations professionnelles qui s'y nouent. En France, comme ailleurs en Europe et dans le monde, la négociation collective se déploie de manière croissante au niveau de l'entreprise, en complément, voire comme substitut à des niveaux sectoriels et nationaux (Jobert et al., 2017 ; Béthoux \& Mias, 2021). L'analyse des négociations d'entreprise - au cœur de réformes récentes dans les pays européens - implique ainsi de s'interroger sur l'existence et les contours de l'entreprise dans un jeu complexe d'externalisation, de filialisation et de sous-traitance qui brouille l'identité, les frontières et les missions de l'entreprise. Cette analyse se situe au sein de dispositifs juridiques ouvrant des espaces sensiblement différents : si, en Amérique du Nord, la négociation part des « unités de négociation » (bargain units) où s'organisent des syndicats dans les établissements, en France, les «conventions d'entreprises » peuvent se conclure à des niveaux variables, depuis celui du groupe dans son intégralité jusqu'à celui de l'établissement. Or, ces accords d'entreprise sont fréquemment appréhendés de manière négative : ni accords de branche, ni accords interprofessionnels, ils sont vus comme l'expression d'un mouvement de décentralisation en tenant pour évidente l'assimilation de l'entreprise à une unité élémentaire de production. Face à cette évidence, il s'agit donc de s'interroger sur les périmètres multiples de l'entreprise, tant dans une définition légale de la convention d'entreprise assimilant accords de groupes, d'entreprises-sociétés et d'établissements, que dans le cas des salariés en soustraitance, mis à disposition ou encore en prestation de service - c'est-à-dire tous les travailleurs et travailleuses qui concourent à l'activité d'une entreprise sans que leur contrat de travail ne les attache à celle-ci comme employeur. Tous ces salariés appartiennent-ils à une même collectivité de travail ? Ne faut-il pas voir dans cette collectivité de travail une entreprise «dissimulée » derrière la multitude de ces cadres juridiques (sociétés, soustraitance et prestation de service) ?

Dès lors, ces négociations dites «d'entreprise » comme notamment les négociations annuelles obligatoires, ou encore celles qui accompagnent les restructurations (Didry, Jobert, 2010 ; Dupuy, 2010), interrogent les missions, les contours et les formes de l'entreprise. De ce point de vue, on peut penser que la définition de l'entreprise passe par les pratiques des acteurs des relations professionnelles saisis non seulement à travers leurs échanges dans des institutions représentatives du personnel, mais aussi par leur participation à la négociation d'accords collectifs et leur confrontation dans des conflits sociaux. Cette réflexion a, par exemple, été au cœur d'un récent rapport pour la DARES (Didry \& Giordano, 2021) dont l'objectif était d'appréhender l'entreprise à travers la pluralité des niveaux (et des lieux) de négociation, dans une tension entre les prérogatives de l'employeur et l'ancrage des collectivités sur les lieux de travail. Ce numéro spécial fait ainsi le pari que l'entreprise peut être envisagée plus spécifiquement à l'aune des négociations collectives qui s'y déroulent. Il entend explorer la manière dont la négociation collective (son périmètre, ses objets, ses résultats) redessine, ou non, les contours de l'entreprise comme ensemble organisé de collectivités de travail révélé 
par le réseau des règles ainsi produites. Pour ce faire, ce numéro spécial articule sept contributions issues de plusieurs disciplines (droit, philosophie, sociologie et relations industrielles) et s'organise en trois axes contributifs.

\section{Négociations et participations des salariés, un enjeu de démocratie dans l'entreprise}

Dans son premier axe, le numéro spécial envisage la morphologie de la négociation d'entreprise que dessine son architecture juridique en France. Si les réformes récentes (loi «travail » de $2016^{7}$ et ordonnances de 2017) mettent l'accent sur la négociation d'entreprise, c'est en s'inscrivant dans le cadre plus général d'un droit du travail explicitant le droit fondamental - établi par l'article 8 du Préambule de la Constitution - de tout travailleur à participer «par l'intermédiaire de ses délégués, à la détermination des conditions de travail ainsi qu'à la gestion de l'entreprise ». En ce sens, le droit dessine une forme de « démocratie au travail » dans l'entreprise (Bisignano et al., 2018) que les réformes n'ont pas éliminé en renvoyant à la collectivité des travailleurs et travailleuses que suppose la désignation de délégués, élus ou syndicaux.

C'est autour de cet enjeu de la démocratie de l'entreprise que se déroule le Grand Entretien que propose ce numéro spécial, réalisé avec Isabelle Ferreras par Élodie Béthoux et Camille Dupuy. Depuis une décennie, Isabelle Ferreras propose une nouvelle organisation de l'entreprise qui permette d'en reconnaitre la pluralité des acteurs et leur légitimité à gouverner en instaurant une gouvernance composée de deux chambres: l'une représentant les travailleurs et travailleuses (les apporteurs en travail), l'autre représentant les actionnaires (les apporteurs en capitaux). Dans cet entretien, Isabelle Ferreras revient sur cette idée structurante de son modèle et explore le rôle de la négociation collective dans cette entreprise démocratique. Elle montre ainsi l'horizon d'une entreprise qui, loin d'être rabattue sur la forme juridique de la société, intègre en plein les travailleurs et travailleuses. Face à une complexification des collectivités de travail, renvoyant tant à la filialisation dans un groupe, qu'à des formes de sous-traitance, de détachements des salariés dans d'autres entreprises que celle de leur employeur, c'est aussi à une véritable redéfinition de l'entreprise qu'appelle cette analyse, par rapport aux cadres fixés par le droit des sociétés.

La contribution de Simon Fouquet s'inscrit elle aussi dans une réflexion sur le partage des intérêts au sein de l'entreprise. Dans une perspective de philosophie du droit, l'auteur met en regard le droit du travail et le droit des sociétés en plaçant la notion d'intérêt au cœur de son analyse. En envisageant la décentralisation de la négociation collective menant de la branche à l'entreprise, telle que la promeuvent les réformes récentes, l'auteur s'interroge ainsi sur un «intérêt de l'entreprise », pouvant apparaître comme une forme d'intérêt général auquel contribueraient les parties à la négociation, syndicats et employeurs, comme porteurs des intérêts particuliers de leurs mandants. Il montre alors que les dernières réformes de la négociation d'entreprise envisagent l'intérêt des salariés comme la somme d'intérêts égoïstes,

\footnotetext{
${ }^{7}$ Loi n $2016-1088$ du 8 août 2016 relative au travail, à la modernisation du dialogue social et à la sécurisation des parcours professionnels.
} 
voire « corporatifs », perdant en cela de vue l'intérêt général de cette entreprise dont ils tirent leur subsistance. Les accords de compétitivité apparaissent emblématiques de cette mise en exergue de l'« égoïsme » de salariés en refusant les concessions consenties par des accords conclus en regard du redressement, du maintien, voire du développement de la rentabilité de l'entreprise et, à terme, de sa pérennité.

Entre l'horizon d'une démocratisation et le constat d'un renforcement de l'intérêt des actionnaires, de quelle entreprise traite alors la négociation d'entreprise ? Loin de s'en tenir à l'évidence d'une négociation d'entreprise par rapport à une négociation de branche dont le législateur entend limiter les prérogatives, il reste à s'interroger sur l'ambivalence fondamentale de cette entreprise dans laquelle ce dernier entend promouvoir la négociation. C'est à cette interrogation que le texte d'Elsa Peskine s'attache à répondre. Selon l'autrice, il se dessine un mouvement historique dans la législation, où une première ouverture tient à la possibilité de négocier des conventions d'établissement assimilées de longue date par le législateur à la «convention d'entreprise ». Certes, la loi du 13 novembre 1983 entendait donner la priorité à l'accord d'entreprise proprement dit, mais la jurisprudence récente en matière d'égalité de traitement a conduit à réaffirmer la portée de la négociation d'établissement. De plus, les réformes de 2016 et 2017 ont intégré dans la catégorie de la « convention d'entreprise », au-delà de ces deux niveaux de l'établissement et de l'entreprise, les accords de groupe. La «porosité » de ces périmètres pour produire des normes du travail témoigne pour l'autrice d'une diversification des possibilités offertes aux partenaires sociaux pour décider ce qui constitue concrètement l'entreprise.

\section{Une démocratie professionnelle au-delà de l'entreprise ?}

Prenant acte de cette grande variabilité dans les définitions de l'entreprise, un deuxième axe du dossier propose d'analyser la manière dont l'entreprise prend concrètement consistance par la négociation, en déplaçant le regard au-delà de l'entreprise à travers la tension entre deux pôles : celui des très grands groupes internationaux, d'une part, et, d'autre part, celui de la branche du cinéma et de l'audiovisuel qui s'avère étonnamment résiliente pour produire un encadrement des conditions de travail dans de très petites entreprises qui se font et se défont selon des projets.

Un premier texte fait le pari de saisir l'« épaisseur sociale » de l'entreprise en partant de son niveau le plus large, et le plus complexe, celui du groupe international ou «entreprise monde », saisie à partir des « accords mondiaux ». Arnaud Mias analyse ces accords conclus à l'échelle de groupes mondiaux qui dépassent les systèmes nationaux de relations professionnelles, établis à partir de négociations menées par des acteurs globaux, directions de groupe et organisations syndicales internationales. L'auteur montre comment ces accords, qui définissent des principes communs à l'ensemble des salariés dans le monde, permettent le déploiement d'une politique sociale spécifique tenant compte de la singularité de chaque groupe étudié. Ces accords apparaissent alors comme des leviers importants pour engager un approfondissement de la connaissance des filiales par les représentants du personnel, en suscitant l'élaboration de ce que l'on pourrait nommer des «bases informationnelles» 
préalable à une coordination des implantations syndicales nationales en vue de développer des formes globales et territorialisées de démocratie salariale.

Cette analyse concrète de la consistance de l'entreprise à partir des négociations est également au cœur de la démarche suivie par Samuel Zarka. Dans le secteur du cinéma, l'entreprise est fondamentalement instable et fluide, se faisant et se défaisant au gré des projets et des productions. Partant du double constat de l'absence d'entreprise stable et de la centralité de la négociation de branche dans le cinéma, il montre comment cette négociation de branche fixe des grilles de qualifications et des conditions de travail à partir desquelles s'engagent des projets prenant la forme d'entreprises relativement éphémères. L'analyse de ces négociations professionnelles lui permettent ainsi de caractériser l'entreprise dans ce secteur. Finalement, cette définition de l'entreprise-projet à partir des cadres de la branche éclaire ce «nouvel esprit du capitalisme» que Luc Boltanski et Ève Chiapello (1999) analysent à travers l'émergence de la «cité par projet», mais ici en s'appuyant sur le droit du travail et la normativité de la branche qui paraissaient assignés à une « cité industrielle».

\section{Les enjeux du conflit dans l'entreprise}

Enfin, un troisième axe du dossier propose d'analyser plus avant l'affrontement des stratégies de ceux et celles qui composent concrètement cette entreprise, les salariés d'un côté, les employeurs de l'autre. C'est ainsi dans la «morphologie » des relations professionnelles que dessinent les législations organisant la négociation collective et la structuration de l'employeur à une échelle multinationale, que se logent les conflits sociaux envisagés tout autant sous la forme des décisions offensives de l'employeur, que sous celle de mobilisations collectives des travailleurs et travailleuses.

C'est du côté de l'analyse des employeurs que le texte Patrick Jalette et Ruth Barton se place. L'auteur et l'autrice mettent au jour les stratégies patronales visant à faire varier les contours de l'entreprise pour contourner les impératifs de négociation collective et réduire l'influence des organisations syndicales. Ils explorent ainsi comment se déploie un avantage stratégique des directions de groupes multinationaux pour imposer des décisions condamnant des acteurs syndicaux ancrés dans l'établissement à se placer sur une position défensive. En effet, les deux entreprises internationales observées jouent le dumping social pour affaiblir l'influence syndicale ancrée dans les établissements de Tasmanie. Leur principal levier est la comparaison des performances économiques entre les établissements, non seulement au niveau australien, mais aussi à une échelle plus vaste en intégrant les implantations néozélandaises. Cela oriente la négociation collective en faveur de l'employeur, en jouant sur la flexibilisation du temps de travail et la modération salariale, qui conduit pour les établissements observés à une hausse de la performance. Dans le même temps, la dimension internationale limite encore la capacité syndicale à s'imposer dans la négociation par la grève en paralysant des établissements clés dans le processus productif. Il devient dès lors difficile pour les salariés de résister à une délocalisation progressive des activités, en plaçant les syndicats sur le terrain des indemnités de départ, sans pouvoir arriver à une coordination efficace avec les syndicats néo-zélandais. 
Compte tenu de l'ouverture laissée, en France, à la négociation d'entreprise entre les niveaux de l'établissement, de l'entreprise et du groupe, Juan Sebastian Carbonell insiste pour sa part sur les stratégies syndicales. L'auteur propose ainsi de rendre compte des correspondances entre niveaux de la négociation (plus ou moins centralisée au sein de l'entreprise) et conflictualité sociale. Ses analyses mettent en avant les marges de manœuvre dont disposent les représentants des personnels pour jouer autour des périmètres de l'entreprise afin de monter des actions collectives aux différentes échelles et de peser in fine dans les négociations. Trois cas du secteur automobile sont ainsi mobilisés. Dans le premier, il souligne l'importance du conflit social pour appuyer la position syndicale dans des négociations annuelles obligatoires se déroulant principalement au niveau de l'établissement. Inversement, dans un deuxième cas, la centralisation de la négociation au niveau du groupe dont témoigne la succession d' « accords de compétitivité » réduit, sans la faire disparaître, les marges d'action syndicale au niveau de l'établissement en matière de mobilisation collective. C'est, enfin, une forme de conflit plus radical qui se manifeste dans un troisième cas.

\section{Pluralité des négociations, des espaces et des niveaux d'analyse}

Dans ce numéro spécial, la négociation collective d'entreprise est donc explorée dans sa diversité. Les négociations collectives étudiées portent sur l'organisation, les conditions du travail et plus généralement sur la vie collective et la gouvernance dans l'entreprise. Ces analyses ont lieu à différentes échelles (de l'acteur mondial, à l'entreprise très localisée) et dans différents secteurs, relèvent de différentes disciplines et s'appuient sur une diversité empirique (entretiens, monographies d'entreprise, analyses d'accords collectifs et d'actes juridiques).

Trois contributions entrent par le prisme d'entreprises mondiales et analysent, en mobilisant différentes approches, les interactions entre siège et filiales. Dans une dynamique de globalisation et plus particulièrement d'européanisation des acteurs économiques, l'entreprise peut ainsi devenir le lieu de négociations collectives transnationales. Alors que les négociations ne sont pas obligatoires au sein des groupes internationaux à une échelle internationale, certains groupes mettent en place des espaces délibératifs au niveau mondial (Edwards et al., 2016) qui conduisent à l'adoption d'accords-cadres mondiaux ou européens (Hennebert \& Bourque, 2010). Ce sont sur ces textes que porte la contribution d'Arnaud Mias qui part du niveau de négociation le plus élevé, celui du groupe, pour analyser comment celuici cadre les niveaux inférieurs (les filiales). Il donne ainsi à voir les politiques d'entreprises monde de dix groupes européens qu'il explore à partir de leurs accords (10 accords) et d'entretiens conduits avec les négociateurs. Dans une perspective inverse, les contributions de Patrice Jalette et Ruth Barton d'un côté, et de Juan Sebastian Carbonell de l'autre, partent du niveau localisé de la filiale. En effet, la politique du groupe peut être perçue comme concurrente à l'égard de l'entreprise inscrite dans un cadre légal et conventionnel établi au sein d'un État. Se pose alors la question du périmètre pertinent au sein duquel peuvent ou non se déployer les rapports entre pouvoir et contre-pouvoir. L'enquête de Patrice Jalette et Ruth Barton nous conduit ainsi en Tasmanie au sein de filiales de deux entreprises multinationales 
(une canadienne, une états-unienne) du secteur manufacturier. Il et elle y ont suivi pendant près de dix ans les négociations collectives autour des questions de rémunération. Leur dispositif méthodologique est précieux en ce qu'il permet une comparaison fine entre différentes stratégies en ayant choisi deux entreprises du même secteur, de la même région et avec le même syndicat. C'est également au sein de filiales de groupes qu'enquête Juan Sebastian Carbonell. À partir d'une démarche monographique qui l'a conduit à analyser les mobilisations collectives et les conflits autour de l'organisation et des conditions du travail au sein de trois établissements du secteur automobile, il analyse les moments de conflits comme des moments particuliers de la négociation collective.

A côté de cette entrée par l'entreprise, trois autres contributions reposent sur l'analyse des textes et des règles de la négociation et de l'entreprise. Dans celles-ci, l'enjeu n'est pas tant d'analyser le contenu des négociations mais de comprendre ce qui préside aux règles d'organisation de la gouvernance d'entreprise et plus largement à la manière dont s'organise la distribution des pouvoirs entre ses parties prenantes. Le travail d'Elsa Peskine comme celui de Simon Fouquet s'appuient ainsi sur l'analyse des textes réglementaires encadrant la négociation. En procédant d'une analyse historique, elle et il montrent comment les conceptions de l'entreprise évoluent au fil de ces textes. Les propos d'Isabelle Ferreras, recueillis par Elodie Béthoux et Camille Dupuy, ont davantage une visée prospective pour dessiner les contours d'une entreprise résolument démocratique. Cependant, dans ce texte comme dans les deux précédents, il s'agit bien pour l'auteur et les autrices de rendre compte de leurs résultats en partant d'exemples concrets de négociations collectives. Ces textes analysent ainsi des dispositifs concrets de négociation. Par exemple, Simon Fouquet analyse en détail les accords de performance collective mis en place lors des dernières réformes du Code du Travail (2017). Isabelle Ferreras pour sa part se penche notamment sur la loi pour la croissance et la transformation des entreprise (PACTE) votée en France en 2019 qui réforme le droit des sociétés en introduisant un statut nouveau d' "entreprise à mission » par l'insertion d'une « raison d'être » dans les statuts de la société qui interroge sur l'ontologie de ce «être ».

Enfin, une dernière contribution fait le pari d'entrer par la branche, dans un dossier qui entend se centrer sur le niveau de l'entreprise et analyser les articulations - non pas classiquement des niveaux de négociation (entreprise, branche, interprofessionnel) (Delahaie et al., 2021) mais des niveaux de l'entreprise elle-même, du plus central (le groupe financier) au plus local (l'établissement). Samuel Zarka explore ainsi en détail la négociation d'une convention collective de branche dans le secteur audiovisuel à partir d'observations des réunions de négociations et de nombreux entretiens avec les négociateurs. Son entrée originale par la branche lui permet de rendre compte de la faible consistance de l'entreprise dans ce secteur.

\section{Saisir l'épaisseur sociale de l'entreprise au travers des négociations}

Ce numéro spécial de la revue Négociations propose ainsi, à travers ces sept contributions explorant différents enjeux, espaces et niveaux, de renouveler les perspectives sur l'entreprise 
en rendant compte de son caractère polysémique d'une part, mais également des stratégies dont cette polysémie est le support.

L'analyse de la structuration légale et conventionnelle, ainsi que du contenu des négociations permet de dégager des éléments qui président aux règles d'organisation de la gouvernance d'entreprise, en éclairant la manière dont se construit la distribution des pouvoirs entre les représentants des travailleurs et travailleuses et ceux des directions. Il s'agit alors de déterminer qui est à l'intérieur et qui est à l'extérieur de l'entreprise, qui concourt au bien commun dont entend se revendiquer l'entreprise et qui, sur cette base, peut prétendre à bénéficier de ses résultats. Les négociations autour de la participation salariée (Crifo \& Rebérioux, 2019), des pouvoirs conférés aux salariés dans les conseils d'administration en envisageant les entreprises comme des «entités politiques » (Ferreras, 2017), ou des autres parties prenantes dans le cadre de la responsabilité sociale des entreprises, sont autant de moments de délibérations sur les contours de l'entreprise et ses acteurs. Mais, loin de s'en tenir à un modèle général de l'entreprise, cet enjeu de démocratisation de l'économie que porte en lui le droit du travail contribue à dessiner des profils spécifiques d'entreprise à partir de l'articulation entre des niveaux d'organisation allant du lieu du travail à l'ensemble des « unités légales » qui composent les groupes.

Loin de se limiter à une relation duale entre syndicats et directions oscillant entre conflit et dialogue social, ce numéro spécial suggère enfin d'envisager des négociations articulant une pluralité de niveaux tant du côté employeur que du côté salarié. Cela conduit à envisager les degrés d'autonomie de ces niveaux, sans consacrer systématiquement la domination du groupe sur les filiales et les établissements. Les conflits collectifs - bien que soumis à une pression expliquant pour une part leur déclin $-\mathrm{y}$ trouvent leur place, comme expression d'une délibération et d'une mobilisation des salariés prolongeant la formulation de revendications. En ce sens, ce dossier invite à considérer, dans leur diversité et leurs dynamiques, ces acteurs qui détiennent le pouvoir de fixer les contours de l'entreprise en prenant part à la négociation collective pour analyser comment ils participent in fine et in concreto à une épaisseur sociale de l'entreprise.

\section{Références}

Barraud de Lagerie, P., 2011, «L'affaire Spectrum: La « responsabilité sociale des entreprises » à l'épreuve d'un drame », Revue française de sociologie, 52, 245-275.

Benedetto-Meyer M., Boboc A., 2021, Sociologie du numérique au travail, Malakoff, Armand Colin.

Béthoux É., Didry C., Mias A., 2007, «What Codes of Conduct Tell Us : corporate responsability and the nature of the multinational firm », Corporate Governance, 15-1, p. 77 90. 
Béthoux É., Mias A., 2021, "How does State-led decentralization affect workplace employment relations? The French case in a comparative perspective", European Journal of Industrial Relations, 27 (1), 5-21

Berle A., Means G., 1932, The Modern Corporation and Private Property, Transactions Publisher.

Bisignano M., Denis J.-M., Dupuy C., Mias A., 2018, « La démocratie au travail : un faux consensus ? », Socio-économie du travail 4, 15-25.

Coase R., 1937, « The nature of the firm”, Economica, 4-16, 386-405.

Crifo P., Rebérioux A., 2019, La participation des salariés, Paris, Presses de Sciences Po.

Delahaie N., Fretel A., 2021, Vers un basculement de la branche vers l'entreprise ? Diversité des pratiques et pluralité des formes d'articulation entre entreprise et branche, Rapport pour la DARES, en ligne.

Didry C., Jobert A., 2010, L'entreprise en restructuration, Dynamiques institutionnelles et mobilisations collectives, Rennes, Presses universitaires de Rennes.

Didry C., Giordano D. (dir.), 2021, Entreprises en négociations. L'entreprise à la lumière des relations professionnelles, Rapport pour la DARES, en ligne.

Didry C., 2020, «L'institution de l'entreprise », Savoir/Agir, 54-4, p. 15-21.

Dupuy C., 2010, « L'entreprise de presse en conflit. Libération et Le Monde en restructuration », Travail et Emploi 124, 29-42.

Edwards T., Sanchez-Mangas R., Jalette P., Lavelle J., Minbaeva D., 2016, "Global Standardization or National Differentiation of HRM Practices in Multinational Companies? A Comparison of Multinationals in Five Countries", Journal of International Business Studies 47(8), 997-1021.

Favereau O. (dir.), 2016, Penser le travail pour penser l'entreprise, Paris, Presses des Mines.

Ferreras I., 2017, Firms as Political Entities. Saving Democracy through Economic Bicameralism, Cambridge University Press.

Hennebert, M., Bourque, R., 2010, «Mondialisation et négociation sociale dans les entreprises multinationales : la négociation de l'accord mondial Quebecor World», Négociations, 14(2), 5-20.

INSEE, 2019, «Les entreprises en France», INSEE Référence, en ligne: https://www.insee.fr/fr/statistiques/4256020.

Jeammaud A., Kirat T., Villeval M.-C., 1996, «Les règles juridiques, l'entreprise et son institutionnalisation : au croisement de l'économie et du droit », Revue internationale de droit économique, 99-140.

Jobert A., Combrexelle J.-D., Legrand H.-J., 2017, « Loi et négociation collective : un débat (encore) d'actualité », Négociations 28 (2), 73-98. 
Kirat T., 2020, « L'entreprise, le nouveau constitutionnalisme et les pouvoirs privés. Nouveaux regards (critiques) sur l'entreprise et le droit », Chassagnon V., Dutraive V. (dir.), Économie politique institutionnaliste de l'entreprise. Travail, démocratie et gouvernement, Paris, Garnier, 287-301

Lyon-Caen A., 2014, «Le droit sans l'entreprise », in Segrestin B., Roger B., Vernac S. (éd.), L'entreprise: Point aveugle du savoir, Auxerre, Éditions Sciences Humaines, 21-31.

Sainsaulieu R. (dir.), 1990, L'entreprise, une affaire de société, Paris, Presses de Sciences Po.

Segrestin D, 1996, Sociologie de l'entreprise. Paris, Armand Colin.

Segrestin B., Hatchuel A., 2012, Refonder l'entreprise, Paris, Seuil.

Segrestin B., Roger B., Vernac S. (éd.), 2014, L'entreprise: Point aveugle du savoir, Auxerre, Éditions Sciences Humaines.

Thuderoz C., 1997, Sociologie des entreprises, Paris, La Découverte.

Weber M., 2003 [1905], L'éthique protestante et l'esprit du capitalisme, traduction J.-P. Grossein, Paris, Gallimard.

Weber M., 2016, Concepts fondamentaux de sociologie, textes choisis, traduits et introduits par Jean-Pierre Grossein, Paris, Gallimard.

Williamson O., 1975, Markets and hierarchies. Analysis and Antitrust Implications, New York, Mac Millan. 